\title{
Tumor immunotherapy-the potential of epigenetic drugs to overcome resistance
}

\author{
Camilla M. Grunewald, Wolfgang A. Schulz, Margaretha A. Skowron, Michèle J. Hoffmann, Guenter \\ Niegisch
}

Department of Urology, Medical Faculty, Heinrich-Heine-University, Duesseldorf, Germany

Contributions: (I) Conception and design: CM Grunewald, WA Schulz, MJ Hoffmann, G Niegisch; (II) Administrative support: G Niegisch, WA Schulz; (III) Provision of study materials or patients: None; (IV) Collection and assembly of data: CM Grunewald; (V) Data analysis and interpretation: All authors; (VI) Manuscript writing: All authors; (VII) Final approval of manuscript: All authors.

Correspondence to: PD Dr. med. Guenter Niegisch. Department of Urology, Medical Faculty, Heinrich-Heine-University, Moorenstraße 5, 40225

Duesseldorf, Germany. Email: guenter.niegisch@med.uni-duesseldorf.de.

\begin{abstract}
Immune checkpoint inhibitors recently introduced into clinical practice have enriched available treatment options for a number of solid cancers. The efficacy of these treatments may however be impaired by tumor cell-intrinsic factors and tumor cell-extrinsic factors resulting in repressed tumor immunogenicity and host immune response. Accordingly, only a subgroup of patients responds to this treatment. Epigenetic drugs may offer an exciting novel approach to reverse immune suppression and to 'prime' tumors for immunotherapy, as DNA methyltransferase (DNMT) inhibitors, histone deacetylase (HDAC) inhibitors and bromodomain and extra-terminal motif (BET) inhibitors show immunomodulatory properties by affecting both cancer cells directly as well as the tumor microenvironment. Upregulation of tumor associated antigens (TAAs), ligands for natural killer (NK) cell receptors, chemokine expression, altered immune checkpoint molecules, antigen procession and presentation as well as induction of viral mimicry improve recognition of cancer cells. In the tumor microenvironment, suppression of myeloid-derived suppressor cells (MDSCs), modulation of regulatory $\mathrm{T}$ cells function and activation of NK cells may improve immunogenic efficacy. In conclusion, current preclinical data supports translation of combinatorial treatment approaches into clinical trials and practice.
\end{abstract}

Keywords: Cancer; immunotherapy; epigenetic modifiers; immune checkpoint inhibitors; combination therapy

Submitted Mar 20, 2018. Accepted for publication Jun 27, 2018.

doi: $10.21037 /$ tcr.2018.06.24

View this article at: http://dx.doi.org/10.21037/tcr.2018.06.24

\section{Introduction}

Only recently, a new class of immunotherapeutic drugs have been introduced for cancer treatment. In brief, these so-called immune checkpoint inhibitors have the ability to restore the antitumor immune response by inhibiting regulatory pathways that turn off the immune response and help cancer cells to evade immune control and elimination especially by cytotoxic T-cells, a process that has been termed "tumor immune evasion" (1).

Amongst others, mechanisms of tumor cell escape include up-regulation of negative co-signals which prevent effective T-cell activation. Therefore, most cancer immuneoncological therapies aim at restoring T-cell-mediated anti-tumor activity $(2,3)$. Despite the promising results of immune checkpoint inhibition many questions remain as only $25 \%$ of the patients respond. A main goal of current research is to expand the number of patients that benefit from immune checkpoint inhibitors. To that end, tumor cell-intrinsic factors, like loss of tumour antigen expression and alterations of interferon and cytokine signalling pathways, as well as factors in the tumour microenvironment (TME), like immune-suppressive cell populations and secreted factors, need to be addressed (4). 
Recent studies have shown that using epigenetic drugs may offer an exciting novel approach to reverse immune suppression and to 'prime' tumors for immunotherapy $(5,6)$. Among these, one study in particular has attracted attention in which 5 patients with advanced non-small cell lung cancer (NSCLC) who progressed after epigenetic therapy were enclosed in a trial for immune checkpoint therapy $(1,7,8)$. In general, $20 \%$ of all NSCLC patients in the trial responded to immune checkpoint therapy, whereas all 5 patients with previous epigenetic therapy passed 24 weeks without progression and three of them developed durable sustained partial responses according to RECIST criteria $(1,9,10)$.

This review aims to summarize our current knowledge on the underlying key mechanisms of epigenetic drugs that improve tumor recognition by cytotoxic $T$ lymphocytes and natural killer (NK) cells and sensitivity to their effector functions, thereby making epigenetic drugs an interesting combination partner for immunotherapy.

\section{Epigenetic changes as pharmacological targets in cancer}

Generally, the term epigenetics is used to describe "heritable and potentially reversible changes in gene expression that occur without altering the DNA sequence" (11-13). Epigenetic changes also help to regulate gene activation in response to extracellular signals. DNA methylation catalysed by DNA methyltransferases (DNMTs) and histone acetylation and deacetylation catalysed by histone acetyltransferases and histone deacetylases (HDACs), respectively, are prototypic epigenetic mechanisms and are often disturbed in cancer. For instance, DNA hypermethylation and histone hypoacetylation lead to silencing of key genes contributing to the neoplastic phenotype (14). Interestingly, several genes inactivated by hypermethylation and/or hypoacetylation are involved in pathways important for immune recognition (15). Consequently, epigenetic drugs targeting DNMTs and HDACs, i.e., DNMT inhibitors (DNMTis) and HDAC inhibitors (HDACis), can be used to upregulate expression of immune signalling components thereby improving tumor cell recognition. More recently, bromodomain and extra-terminal motif (BET) proteins have been discovered as epigenetic targets in tumor therapy, as some are overexpressed in various cancer types. They too seem to be involved in the regulation of the programmed death-1 (PD-1)/programmed death ligand-1 (PD-L1) immune checkpoint axis making BET inhibitors another interesting combination partner for checkpoint inhibitors (16).

\section{DNMTis}

Based on their molecular structure, two major classes of DNMTis are distinguished, cytidine nucleoside analogs and non-nucleosides. Nucleoside analogs include 5-azacytidine (azacytidine, AZA), 5-aza-2'-deoxycytidine (decitabine, DAC), 5-fluoro-2'-deoxycytidine, 5,6-dihydro5 -azacytidine and zebularine (11) with azacytidine and decitabine being used for the treatment of myelodysplastic syndrome (MDS) as well as acute myeloid leukaemia (AML) $(17,18)$. Second generation nucleoside analogs include the dinucleotide of decitabine and deoxyguanosine, SGI-110, with improved pharmacokinetic properties. Following uptake and phosphorylation to nucleotides these cytidine analogs are incorporated into genomic DNA of replicating cells. When DNMTs attempt to methylate them, the enzymes become irreversibly inactivated. Through this mechanism newly synthesized DNA strands cannot be methylated leading to passive demethylation of the genome following DNA replication $(11,15,19)$.

Non-nucleoside analogs on the other hand show hypomethylating activity without being incorporated into DNA. They can be divided into synthetic small molecules (e.g., hydralazine, procainamide and RG108), natural molecules [epigallocatechin-3-gallate (EGCG)] and oligonucleotides (MG98, mir-29b), each with different mechanisms of action (11)

\section{HDAC inhibitors}

Classical HDACs are usually subdivided into four classes. Class I comprises HDAC1, 2, 3 and 8, class IIA HDAC 4, 5, 7, and 9, class IIB HDAC 6 and 10, and class IV HDAC11 (20). They share $\mathrm{Zn}^{2+}$-dependent catalytic activity; class I enzymes are expressed in the nucleus, class IIB in the cytoplasm and class IIA shuttle between cytoplasm and nucleus (21-23).

Compounds targeting these enzymes are commonly referred to as HDACis (11). Depending on their chemical structure they are classified into short-chain and aromatic fatty acids (e.g., sodium butyrate, 4-phenylbutyrate and valproic acid), hydroxamic acids (e.g., trichostatin A), hydroxamic acid-based hybrid polar compounds (e.g., SAHA (vorinostat) and panobinostat), cyclic tetrapeptides (e.g., romidepsin), benzamides (e.g., SNDX-275 and mocetinostat) 
and miscellaneous compounds like depudecin (24).

In general, benzamide class HDACis target class I HDAC isoforms whereas hydroxamic acid class HDACis target both, class I (HDAC1, 2, and 3) and class IIB (HDAC6) $(25,26)$. Until today, vorinostat and romidepsin have been approved for treatment of cutaneous $T$ cell lymphoma, belinostat and chidamide for peripheral $\mathrm{T}$ cell lymphoma and panobinostat for multiple myeloma (27).

\section{BET inhibitors}

BET proteins include BRD2, BRD3, BRD4 and bromodomain testis-specific protein (BRDT). They contain $2 \mathrm{~N}$-terminal bromodomains which preferentially bind to multi-acetylated peptide sequences to stimulate transcription and regulate various cellular functions, including cell cycle progression and cell proliferation (16). In the last years, several BET inhibitors have been developed. JQ1 (a thienotriazolodiazepine) and iBET (a benzodiazepine) were first described in $2010(28,29)$. Both compounds are pan-BET bromodomain inhibitors which discriminate neither between the 2 bromodomains within the same BET protein nor among the 4 BET family members (30). Aiming to improve selectivity, BRD1 selective inhibitors (e.g., MS-436, Olinone and BI-2536) and BRD2 selective inhibitors (e.g., RVX-208 and RVX-297) have been developed as well as further pan inhibitors (30). In tumor therapy, especially inhibition of BRD4 which is thought to cooperate with MYC is considered important. However, none of the currently available BET inhibitors is very selective and these compounds therefore are likely to act by diverse mechanisms (31). Similar to DNMTis and HDACis there is evidence suggesting immunomodulatory functions of BET inhibitors, but especially their specific actions on tumor and immune cells need further exploration.

\section{Epigenetic immunomodulation of cancer cells}

Epigenetic drugs exert direct effects on tumor cells by affecting expression of antigenic proteins, presentation of antigens, immune checkpoints, stress responses, and antiviral signaling pathways (see Figure 1).

\section{Tumor associated antigens (TAAs)}

Immune attraction properties of cancer cells are dependent on expression of TAAs (32). Among these, the large family of cancer testis antigens (CTAs) is well characterized (33). CTAs are expressed in various human tumors but not in normal tissues as they are normally silenced in mature somatic cells primarily by DNA methylation $(27,33)$. Outside the immune privileged testicular tissue, they are recognized by cytotoxic T-lymphocytes (CTLs). CTAs include melanoma-associated antigen (MAGE), preferentially expressed antigen of melanoma (PRAME), New York esophageal squamous cell carcinoma-1 (NYESO-1) and the SSX as well as GAGE/PAGE/XAGE gene families (27). DNMTis can cause increased CTA expression in tumors via promoter demethylation hence leading to improved targeting by the host immune system. For instance, CTAs were significantly upregulated by azacytidine in the majority of 77 epithelial cancer cell lines, most strongly in colorectal (64\%) and ovarian (39\%) cancer lines $(6,9)$. The newer DNMTi SGI-110 induced hypomethylation and CTA gene expression in a dosedependent manner in epithelial ovarian cancer cells and xenografts. The observed effects were superior to those of azacytidine or decitabine (34). In a Phase I clinical trial on patients with relapsed epithelial ovarian cancer, decitabine added to NY-ESO-1 vaccine and doxorubicin chemotherapy elicited DNA hypomethylation at the NY-ESO-1 promoter and upregulation of NY-ESO-1. Moreover, increased serum antibodies to NY-ESO-1 and T-cell responses were observed in the majority of the patients (35). Similarly, chidamide, a selective HDAC1, 2, 3, and 10 inhibitor, was shown to increase PRAME mRNA expression in human acute myeloid leukemia cell lines and primary acute myeloid leukemia cells in vitro (36).

\section{Antigen processing and presentation}

Further, T-cell depletion of neoplastic cells requires their recognition via antigen presentation in the form of antigenic peptides associated with a specific MHC class I molecule on the tumor cell surface $(11,37)$. In order to escape immune recognition, tumor cells often carry specific alterations in MHC class I molecules which impair their identification by CTLs $(11,38)$. Expression of MHC class I molecules in cancer cells is often reduced by epigenetic mechanisms (39). DNMTis and/or HDACis can upregulate MHC class I antigens on different neoplastic cells, including cell lines from neuroblastoma, cervical and prostate cancer (11,40-42). It is unknown to which extent this upregulation occurs in vivo.

Besides MHC class I molecule on the cell surface, 


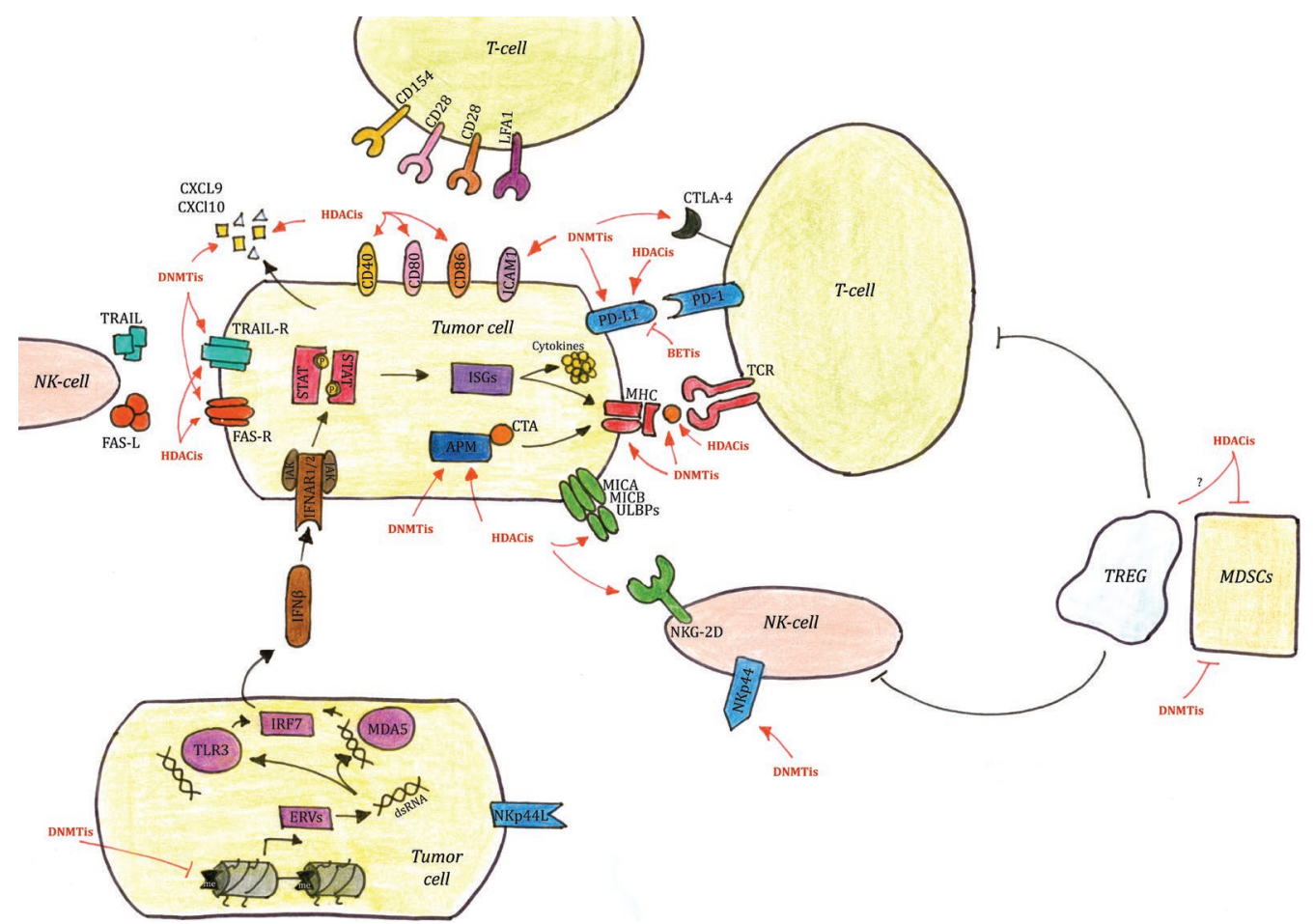

Figure 1 Immunomodulatory properties of epigenetic drugs on cancer cells as well as on the tumor microenvironment (see text for detailed information). HDACis, histone deacetylase inhibitors; DNMTis, DNA methyltransferase inhibitors; BETis, bromodomain and extraterminal motif inhibitors; CTA, cancer testis antigens; APM, antigen processing and presenting machinery; MHC, major histocompatibility complex; ULBPs, UL16 binding proteins; CD, cluster of differentiation; ICAM1, intracellular adhesion molecule 1; LFA1, lymphocyte function-associated antigen 1; PD-1, programmed death receptor-1; PD-L1, programmed death-ligand 1; TCR, T cell receptor; NK cell, natural killer cell; NKG-2D receptor, natural-killer group 2 receptor, member D; TREG, T-regulatory cell; MDSC, myeloid-derived suppressor cell; ERVs, endogenous retroviruses; dsRNA, double-stranded RNA; IFN, interferon; IFNAR, interferon- $\alpha / \beta$ receptor; ISGs, interferon-stimulated genes; FAS-R/-L, first apoptosis signal receptor/ligand; TRAIL (receptor), tumor necrosis factor-related apoptosisinducing ligand (receptor).

expression of other components of the antigen processing machinery (APM) may also be defective in cancer $(43,44)$. Both DNMTis and HDACis have been shown to induce or enhance the expression of different APM pathway components such as TAP-1, TAP-2, LMP2, LMP7 and tapasin in various tumor types (42,44-46).

Moreover, exposure to epigenetic agents can induce surface expression of costimulatory molecules on cancer cells like CD40, CD80, CD86 and ICAM-1 (44). Interestingly, DNMTis and HDACis seem to have different tropisms. Whereas ICAM-1 appears to be mainly targeted by DNMTis, HDACis preferentially upregulate CD40, CD80, and CD86 (11). More precisely, decitabine and its newly derived pro drug SGI-110 have been shown to upregulate ICAM-1 in different cell lines including cutaneous melanoma, mesothelioma, renal cell carcinoma (RCC) and sarcoma $(47,48)$. On the other hand, trichostatin A and sodium butyrate have been shown to enhance cell surface expression of CD40, CD80 and CD86, besides increasing class I and II MHC expression, in various melanoma, neuroblastoma and plasmacytoma cell lines $(27,42,45,49)$.

\section{Upregulation of immune checkpoints}

Another straightforward way by which epigenetic drugs sensitize cancer cells to immune checkpoint therapy is by upregulating the immune checkpoint proteins CTLA-4 (cytotoxic T-lymphocyte-associated protein), PD-1, PD-L1, and PD-L2 on tumor cells and tumor-infiltrating lymphocytes (44). In a murine melanoma model, HDAC inhibition upregulated especially PD-L1 in vitro as well as in vivo, with panobinostat as the most efficient compound (50). 
Similarly, after treatment with decitabine, increased levels of PD-L1 were found in epithelial ovarian cancer and non-small cellular lung cancer cell lines $(6,9)$. Treatment of leukemia cells with decitabine likewise resulted in dosedependent upregulation of PD-L1, PD-L2, PD-1 and CTLA-4 gene expression. Exposure to decitabine resulted in partial demethylation of the PD-1 gene in leukemia cell lines and primary human samples (51).

Unlike HDACis and DNMTis, BET inhibitors seem to inhibit PD-L1 expression showing synergy with immune checkpoint inhibitors. CD274, for example, which encodes PD-L1, is a direct target of BRD4-enhanced gene transcription. In a mouse model of ovarian cancer, treatment with the BET inhibitor JQ1 significantly reduced PD-L1 expression on tumor and immune cells leading to an increased activity of anti-tumor cytotoxic T cells (52). Similarly, in a NSCLC xenograft, JQ1 in combination with anti-PD-1 immune checkpoint blockade decreased tumor burden and improved survival (53). Similar synergistic effects were seen in combination with epacadostat, an indoleamine-2,3-dioxygenase 1 (IDO-1) inhibitor, suggesting that multiple immune checkpoints may interact with BET proteins $(16,54)$.

\section{Stress-inducing ligands and death inducing receptors}

Apart from components of the APM and immune checkpoint proteins, several studies have shown that HDACis may also induce increased expression of NK cell ligands on tumor cells, thereby sensitizing tumor cells to NK cell lysis. Amongst others, HDACis like sodium butyrate, SAHA, MS-275 and VPA upregulated the expression of MICA, MICB and ULBP1-3 in various tumor cells (55-57). Mechanistically, the upregulation resulted from direct effects such as hyperacetylation of histones associated with promoters of NKG2 ligands (57) and indirect effects such as activation of the ATM/ATRdependent DNA damage response (56). Further, there is strong evidence that HDACis, alone or in combination with DNMTis, have the ability to upregulate the expression of FAS and TRAIL death receptors on cancer cells, thereby further enhancing NK cell killing (57-59).

\section{Viral mimicry}

Several recent papers have described activation of antiviral signaling pathways especially by DNMTis, but also to some degree by HDACis. Chiappinelli and coworkers observed increased interferon signaling and concordant upregulation of surface antigens and their assembly proteins in 77 epithelial cancer cell lines treated with decitabine $(1,6)$. They defined a 300-gene expression set, named AzaInduced iMmune genes (AIM) (6). Excitingly, expression of AIM divided primary tumor samples from 'The Cancer Genome Atlas' into high and low expression groups (6). It was therefore hypothesized that decitabine could reverse the immune evasion pattern of low AIM tumors subsequently sensitizing them to immune therapy $(1,6)$.

Subsequently, the same group and Roulois et al. suggested that this immune signaling is mainly activated by induced dsRNAs derived at least in part from endogenous retroviral sequences (ERVs) $(5,60)$. This mechanism is now commonly referred to as viral mimicry.

ERVs, also known as long terminal repeat (LTR) retrotransposons, make up approximately $8 \%$ of the human genome. They derive from ancient retroviral infections and are normally silenced by promoter DNA methylation and other epigenetic mechanisms, but upon reactivation, are capable of eliciting innate and adaptive host immune responses $(44,61)$.

In subsequent work on ovarian and colorectal cancer cell lines, it was shown that DNMTis (azacytidine and decitabine) activate a canonical interferon signaling pathway through upregulation of dsRNA that activates the cytosolic dsRNA sensors TLR3 and MDA5 thereby inducing type I and type III interferons, upregulation of interferon response genes and JAK/STAT signaling $(5,60)$. This cascade involves nuclear translocation of IRF7 and activation of antiviral response programs, including immunogenic cell death for viral clearance $(60,62)$. In essence, DNMTis seem to be able to deceive cancer cells into behaving like virus-infected cells leading to an anti-viral immune response directed towards cancer cells (60). Underlining these results, clinical trials on NSCLC, breast cancer and colorectal cancer with low dose DNMTis also showed upregulation of interferonresponsive genes $(6,9)$.

\section{Epigenetic immunomodulation in the tumor microenvironment}

Epigenetic drugs not only affect cancer cells, but also exert important immunomodulatory effects on other cell types in the tumor microenvironment and paracrine signaling (see Figure 1). 


\section{NK cells}

Like cancer cells, epigenetic drugs also affect and can thereby modulate host immune cells. For example, entinostat was shown to enhance NK cell killing of cancer cells through upregulation of the activating receptor NKG2D on the surface of NK cells in human colon carcinoma and sarcoma cells (63). Similarly, decitabine sensitized AML blasts to lysis by NK cells in vitro via upregulation of killer immunoglobulin-like receptors and the activating receptor NKp44 on NK-cells. Noteworthy, high doses of decitabine decreased NK cell proliferation and viability, suggesting that optimal doses for the beneficial immune effects of epigenetic therapy may be rather low (64).

\section{Suppression of myeloid-derived suppressor cells (MDSCs)}

More recently, there has been growing evidence that epigenetic drugs may have an effect on MDSCs. MDSCs are a type of immature myeloid cells suppressing antitumor immunity. They are induced by tumor growth and accumulate in the TME (27). Their depletion may therefore enhance anti-tumor immunity. HDACis treatment has been reported to decrease MDSC accumulation in the spleen, blood and tumor bed whilst increasing the proportion of $\mathrm{T}$ cells in mice with mammary tumors. The HDACis-induced increase in MDSC apoptosis may be due to increased intracellular reactive oxygen species (65). Similarly, the percentage of MDSCs in the TME and spleens of mice bearing prostate adenocarcinoma or MHC class I-deficient TC-1 tumors decreased following treatment with azacytidine (66).

\section{T regulatory cells (Tregs)}

Similar to MDSCs, CD4+CD25+FoxP3+ Tregs are typically immunosuppressive and contribute to tumor immune tolerance. Under normal conditions, they have an important function in maintaining immune response homeostasis by preventing over-activation and autoimmunity. In the TME, however, Tregs often proliferate in response to factors secreted by tumor or stroma cells and impair antitumor immune responses by inhibiting NK and T-cell function (27).

Expression of FOXP3, which regulates Treg function and development, is tightly regulated by epigenetic mechanisms (67) and is therefore altered by epigenetic therapies. This straightforward approach at improving immunotherapy is however complicated by the fact that opposite mechanisms have been described by which different HDACs affect Treg and Foxp3 expression $(68,69)$. Whereas inhibition of HDAC6 and HDAC9 was shown to even augment the suppressive functions of Tregs (70), targeting HDAC5 in a different study led to reduced suppressive function of Tregs in vitro and in vivo (71).

\section{Induction of chemokine expression}

Another way by which epigenetic modulation can cause tumor immune escape is via epigenetic repression of chemokines needed for immune cell attraction. Epigenetic silencing of chemokine genes may occur by DNMTmediated DNA methylation (72). Accordingly, treatment of ovarian cancer cells with decitabine was shown to slow down tumor progression and to increase effector T-cell tumor infiltration by increasing expression of the $\mathrm{T}$ helper 1-type chemokines CXCL9 and CXCL10. Moreover, the therapeutic efficacy of PD-L1 checkpoint blockade was improved (72). Concordant effects have been seen in tumorbearing mice (72-74).

For HDACis, upregulation of chemokine expression, increased T-cell infiltration and T-cell-dependent tumor regression was elicited by romidepsin with significantly enhanced responses to PD-1 blockade immunotherapy in lung tumor models (75).

\section{Epigenetic immunomodulation in urological tumors}

In patients with advanced or metastatic RCC, the use of nivolumab (anti-PD-1 antibody) has shown improved median overall survival compared to everolimus in a large randomized controlled trial (RCT) (76). Consequently, its use for treatment of patients with metastatic RCC who have previously progressed to one or two regimens of antiangiogenic therapy was FDA (U.S. Food and Drug Administration) and EMA (European Medicines Agency) approved in 2015 and 2016 respectively (77).

For the treatment of patients with locally advanced or metastatic urothelial carcinoma, who failed to respond to prior platinum-containing therapy, two immune checkpoint inhibitors (nivolumab and pembrolizumab, both anti-PD-1 monoclonal antibodies) have been approved following data from corresponding RCTs $(78,79)$. Pembrolizumab has been approved additionally for the treatment of patients who are not eligible for cisplatin-containing chemotherapy (80). Meanwhile, the anti-PD-L1 antibody atezolizumab has also 
obtained FDA and EMA approval for the above indications. Regarding immune checkpoint inhibition in advanced prostate cancer, however, only modest clinical activity has been observed (81).

Whereas the clinical use of immune checkpoint inhibition in urogenital malignancies is thus increasing over the last few years, data on the immunomodulatory effects of epigenetic therapies regarding these tumor entities is currently scarce. In a study investigating the effects of valproic acid and INF- $\alpha$ on gene transcription of metastatic RCC cell lines, the list of upregulated genes also included several chemokines (CXCL10, CXCL11, CXCL16) (82). Similarly, immunomodulatory effects of entinostat on RCC and prostate cancer emerged in a study evaluating Treg function. Here, low dose entinostat reduced Foxp3 levels in Tregs, which was associated with enhanced tumor growth inhibition (69). In a different study prostate carcinoma cells, amongst others, became more sensitive to T-cell mediated lysis in vitro after clinically relevant exposure to either vorinostat or entinostat. Here, a broad range of tumorassociated antigens, such as CEA, MUC1 and PSA, was observed and an association with augmented expression of multiple proteins involved in antigen processing and tumor immune recognition was found (83). Especially in prostate cancer, decreased expression of CTAs has been discovered as a means of escaping immune monitoring, with several studies addressing the role of HDACis and DNMTis (e.g., azacytidine) in increasing tumor immunogenicity $(84,85)$. Although induction of CTAs by azacytidine and trichostatin A was also observed for bladder cancer $(86,87)$, our research did not yield further results regarding immunomodulation through epigenetic therapy in bladder cancer. Thus, especially in the field of uro-oncology, more preclinical research is needed.

\section{Conclusions}

As summarized in Figure 1, epigenetic modifiers such as DNMTis, HDACis and BET inhibitors show immunomodulatory properties both on cancer cells directly as well as on other cells and factors in the tumor microenvironment. In cancer cells, upregulation of TAAs, NK cell ligands, chemokine expression and immune checkpoints, antigen procession and presentation as well as induction of viral mimicry improve recognition and elimination of cancer cells by cytotoxic host cells. In the tumor microenvironment, suppression of MDSCs, modulation of Treg function and activation of NK cells may improve immunogenic efficacy.

In conclusion, current preclinical data supports translation of combinatorial treatment approaches into clinical trials and practice.

\section{Acknowledgments}

Funding: None.

\section{Footnote}

Conflicts of Interest: All authors have completed the ICMJE uniform disclosure form (available at http://dx.doi. org/10.21037/tcr.2018.06.24). The authors have no conflicts of interest to declare.

Ethical Statement: The authors are accountable for all aspects of the work in ensuring that questions related to the accuracy or integrity of any part of the work are appropriately investigated and resolved.

Open Access Statement: This is an Open Access article distributed in accordance with the Creative Commons Attribution-NonCommercial-NoDerivs 4.0 International License (CC BY-NC-ND 4.0), which permits the noncommercial replication and distribution of the article with the strict proviso that no changes or edits are made and the original work is properly cited (including links to both the formal publication through the relevant DOI and the license). See: https://creativecommons.org/licenses/by-nc-nd/4.0/.

\section{References}

1. Chiappinelli KB, Zahnow CA, Ahuja N, et al. Combining epigenetic and immunotherapy to combat cancer. Cancer Res 2016;76:1683-9.

2. Bidnur S, Savdie R, Black PC. Inhibiting immune checkpoints for the treatment of bladder cancer. Bladder Cancer 2016;2:15-25.

3. Romano E, Romero P. The therapeutic promise of disrupting the PD-1/PD-L1 immune checkpoint in cancer: unleashing the CD8 $\mathrm{T}$ cell mediated anti-tumor activity results in significant, unprecedented clinical efficacy in various solid tumors. J Immunother Cancer 2015;3:15.

4. Sharma P, Hu-Lieskovan S, Wargo JA, et al. Primary, adaptive, and acquired resistance to cancer immunotherapy. Cell 2017;168:707-23.

5. Chiappinelli KB, Strissel PL, Desrichard A, et al. 
Inhibiting DNA methylation causes an interferon response in cancer via dsRNA including endogenous retroviruses. Cell 2017;169:361.

6. Li H, Chiappinelli KB, Guzzetta AA, et al. Immune regulation by low doses of the DNA methyltransferase inhibitor 5-azacitidine in common human epithelial cancers. Oncotarget 2014;5:587-98.

7. Topalian SL, Hodi FS, Brahmer JR, et al. Safety, activity, and immune correlates of anti-PD-1 antibody in cancer. $\mathrm{N}$ Engl J Med 2012;366:2443-54.

8. Brahmer J, Reckamp KL, Baas P, et al. Nivolumab versus docetaxel in advanced squamous-cell non-small-cell lung cancer. N Engl J Med 2015;373:123-35.

9. Wrangle J, Wang W, Koch A, et al. Alterations of immune response of Non-Small Cell Lung Cancer with Azacytidine. Oncotarget 2013;4:2067-79.

10. Juergens RA, Wrangle J, Vendetti FP, et al. Combination epigenetic therapy has efficacy in patients with refractory advanced non-small cell lung cancer. Cancer Discov 2011;1:598-607.

11. Sigalotti L, Fratta E, Coral S, et al. Epigenetic drugs as immunomodulators for combination therapies in solid tumors. Pharmacol Ther 2014;142:339-50.

12. Terranova-Barberio M, Thomas S, Munster PN. Epigenetic modifiers in immunotherapy: a focus on checkpoint inhibitors. Immunotherapy 2016;8:705-19.

13. Schulz WA. Integrating epigenetics. Biol Chem 2014;395:1263-4.

14. Baylin SB, Jones PA. Epigenetic determinants of cancer. Cold Spring Harb Perspect Biol 2016;8. doi: 10.1101/ cshperspect.a019505.

15. Sigalotti L, Fratta E, Coral S, et al. Epigenetic drugs as pleiotropic agents in cancer treatment: biomolecular aspects and clinical applications. J Cell Physiol 2007;212:330-44.

16. Doroshow DB, Eder JP, LoRusso PM. BET inhibitors: a novel epigenetic approach. Ann Oncol 2017;28:1776-87.

17. Khan H, Vale C, Bhagat T, et al. Role of DNA methylation in the pathogenesis and treatment of myelodysplastic syndromes. Semin Hematol 2013;50:16-37.

18. Thomas X. DNA methyltransferase inhibitors in acute myeloid leukemia: discovery, design and first therapeutic experiences. Expert Opin Drug Discov 2012;7:1039-51.

19. Stresemann C, Lyko F. Modes of action of the DNA methyltransferase inhibitors azacytidine and decitabine. Int J Cancer 2008;123:8-13.

20. Pinkerneil M, Hoffmann MJ, Niegisch G. Epigenetic treatment options in urothelial carcinoma. Methods Mol
Biol 2018;1655:289-317.

21. Cernotta N, Clocchiatti A, Florean C, et al. Ubiquitindependent degradation of HDAC4, a new regulator of random cell motility. Mol Biol Cell 2011;22:278-89.

22. Clocchiatti A, Di Giorgio E, Demarchi F, et al. Beside the MEF2 axis: unconventional functions of HDAC4. Cell Signal 2013;25:269-76.

23. Pinkerneil M, Hoffmann MJ, Schulz WA, et al. HDACs and HDAC inhibitors in urothelial carcinoma perspectives for an antineoplastic treatment. Curr Med Chem 2017. [Epub ahead of print].

24. Nebbioso A, Carafa V, Benedetti R, et al. Trials with 'epigenetic' drugs: an update. Mol Oncol 2012;6:657-82.

25. Topper MJ, Vaz M, Chiappinelli KB, et al. Epigenetic therapy ties MYC depletion to reversing immune evasion and treating lung cancer. Cell 2017;171:1284-1300.e21.

26. Bradner JE, West N, Grachan ML, et al. Chemical phylogenetics of histone deacetylases. Nat Chem Biol 2010;6:238-43.

27. Mazzone R, Zwergel C, Mai A, et al. Epi-drugs in combination with immunotherapy: a new avenue to improve anticancer efficacy. Clin Epigenetics 2017;9:59.

28. Filippakopoulos P, Qi J, Picaud S, et al. Selective inhibition of BET bromodomains. Nature 2010;468:1067-73.

29. Nicodeme E, Jeffrey KL, Schaefer U, et al. Suppression of inflammation by a synthetic histone mimic. Nature 2010;468:1119-23.

30. Xu Y, Vakoc CR. Targeting cancer cells with BET bromodomain inhibitors. Cold Spring Harb Perspect Med 2017;7. doi: 10.1101/cshperspect.a026674.

31. Andrieu G, Belkina AC, Denis GV. Clinical trials for BET inhibitors run ahead of the science. Drug Discov Today Technol 2016;19:45-50.

32. Heninger E, Krueger TE, Lang JM. Augmenting antitumor immune responses with epigenetic modifying agents. Front Immunol 2015;6:29.

33. Fratta E, Coral S, Covre A, et al. The biology of cancer testis antigens: putative function, regulation and therapeutic potential. Mol Oncol 2011;5:164-82.

34. Srivastava P, Paluch BE, Matsuzaki J, et al. Immunomodulatory action of the DNA methyltransferase inhibitor SGI-110 in epithelial ovarian cancer cells and xenografts. Epigenetics 2015;10:237-46.

35. Odunsi K, Matsuzaki J, James SR, et al. Epigenetic potentiation of NY-ESO-1 vaccine therapy in human ovarian cancer. Cancer Immunol Res 2014;2:37-49.

36. Yao Y, Zhou J, Wang L, et al. Increased PRAME-Specific CTL killing of acute myeloid leukemia cells by either 
a novel histone deacetylase inhibitor chidamide alone or combined treatment with decitabine. PLoS One. 2013;8:e70522.

37. Topfer K, Kempe S, Muller N, et al. Tumor evasion from T cell surveillance. J Biomed Biotechnol 2011;2011:918471.

38. Seliger B. Molecular mechanisms of MHC class I abnormalities and APM components in human tumors. Cancer Immunol Immunother 2008;57:1719-26.

39. Campoli M, Ferrone S. HLA antigen changes in malignant cells: epigenetic mechanisms and biologic significance. Oncogene 2008;27:5869-85.

40. Adair SJ, Hogan KT. Treatment of ovarian cancer cell lines with 5-aza-2'-deoxycytidine upregulates the expression of cancer-testis antigens and class I major histocompatibility complex-encoded molecules. Cancer Immunol Immunother 2009;58:589-601.

41. Kitamura H, Torigoe T, Asanuma H, et al. Downregulation of HLA class I antigens in prostate cancer tissues and up-regulation by histone deacetylase inhibition. J Urol 2007;178:692-6.

42. Magner WJ, Kazim AL, Stewart C, et al. Activation of MHC class I, II, and CD40 gene expression by histone deacetylase inhibitors. J Immunol 2000;165:7017-24.

43. Bukur J, Jasinski S, Seliger B. The role of classical and non-classical HLA class I antigens in human tumors. Semin Cancer Biol 2012;22:350-8.

44. Dunn J, Rao S. Epigenetics and immunotherapy: The current state of play. Mol Immunol 2017;87:227-39.

45. Khan AN, Gregorie CJ, Tomasi TB. Histone deacetylase inhibitors induce TAP, LMP, Tapasin genes and MHC class I antigen presentation by melanoma cells. Cancer Immunol Immunother 2008;57:647-54.

46. Setiadi AF, Omilusik K, David MD, et al. Epigenetic enhancement of antigen processing and presentation promotes immune recognition of tumors. Cancer Res 2008;68:9601-7.

47. Coral S, Sigalotti L, Gasparollo A, et al. Prolonged upregulation of the expression of HLA class I antigens and costimulatory molecules on melanoma cells treated with 5-aza2'-deoxycytidine (5-AZA-CdR). J Immunother 1999;22:16-24.

48. Coral S, Parisi G, Nicolay HJ, et al. Immunomodulatory activity of SGI-110, a 5-aza-2'-deoxycytidine-containing demethylating dinucleotide. Cancer Immunol Immunother 2013;62:605-14.

49. Kalbasi A, Fonsatti E, Natali PG, et al. CD40 expression by human melanocytic lesions and melanoma cell lines and direct CD40 targeting with the therapeutic anti-CD40 antibody CP-870,893. J Immunother 2010;33:810-6.

50. Woods DM, Sodre AL, Villagra A, et al. HDAC inhibition upregulates PD-1 Ligands in melanoma and augments immunotherapy with PD-1 blockade. Cancer Immunol Res 2015;3:1375-85.

51. Yang H, Bueso-Ramos C, DiNardo C, et al. Expression of PD-L1, PD-L2, PD-1 and CTLA4 in myelodysplastic syndromes is enhanced by treatment with hypomethylating agents. Leukemia 2014;28:1280-8.

52. Zhu H, Bengsch F, Svoronos N, et al. BET bromodomain inhibition promotes anti-tumor immunity by suppressing PD-L1 expression. Cell Rep 2016;16:2829-37.

53. Adeegbe DO FG, Wong KK. BET bromodomain inhibition synergizes with immune checkpoint blockade to facilitate anti-tumor response in a murine model of non-small cell lung cancer harboring activating KRAS mutation. The Journal of Immunology 2016;196.

54. Koblish HK HM, Hall L, et al. The BET inhibitor INCB054329 enhances the activity of checkpoint modulation in syngeneic tumor models. Cancer Research 2016;76:4904

55. Armeanu S, Bitzer M, Lauer UM, et al. Natural killer cellmediated lysis of hepatoma cells via specific induction of NKG2D ligands by the histone deacetylase inhibitor sodium valproate. Cancer Res 2005;65:6321-9.

56. Berghuis D, Schilham MW, Vos HI, et al. Histone deacetylase inhibitors enhance expression of NKG2D ligands in Ewing sarcoma and sensitize for natural killer cell-mediated cytolysis. Clin Sarcoma Res 2012;2:8.

57. Yamanegi K, Yamane J, Kobayashi K, et al. Valproic acid cooperates with hydralazine to augment the susceptibility of human osteosarcoma cells to Fas- and NK cell-mediated cell death. Int J Oncol 2012;41:83-91.

58. Yang D, Torres CM, Bardhan K, et al. Decitabine and vorinostat cooperate to sensitize colon carcinoma cells to Fas ligand-induced apoptosis in vitro and tumor suppression in vivo. J Immunol 2012;188:4441-9.

59. Lundqvist A, Abrams SI, Schrump DS, et al. Bortezomib and depsipeptide sensitize tumors to tumor necrosis factor-related apoptosis-inducing ligand: a novel method to potentiate natural killer cell tumor cytotoxicity. Cancer Res 2006;66:7317-25.

60. Roulois D, Loo Yau H, Singhania R, et al. DNAdemethylating agents target colorectal cancer cells by inducing viral mimicry by endogenous transcripts. Cell 2015;162:961-73.

61. Seifarth W, Frank O, Zeilfelder U, et al. Comprehensive analysis of human endogenous retrovirus transcriptional activity in human tissues with a retrovirus-specific microarray. J Virol 2005;79:341-52.

62. Barbalat R, Ewald SE, Mouchess ML, et al. Nucleic acid recognition by the innate immune system. Annu Rev 
Immunol 2011;29:185-214.

63. Zhu S, Denman CJ, Cobanoglu ZS, et al. The narrowspectrum HDAC inhibitor entinostat enhances NKG2D expression without NK cell toxicity, leading to enhanced recognition of cancer cells. Pharm Res 2015;32:779-92.

64. Kopp LM, Ray A, Denman CJ, et al. Decitabine has a biphasic effect on natural killer cell viability, phenotype, and function under proliferative conditions. Mol Immunol 2013;54:296-301.

65. Wang HF, Ning F, Liu ZC, et al. Histone deacetylase inhibitors deplete myeloid-derived suppressor cells induced by 4T1 mammary tumors in vivo and in vitro. Cancer Immunol Immunother 2017;66:355-66.

66. Mikyskova R, Indrova M, Vlkova V, et al. DNA demethylating agent 5-azacytidine inhibits myeloidderived suppressor cells induced by tumor growth and cyclophosphamide treatment. J Leukoc Biol 2014;95:743-53.

67. Morikawa H, Sakaguchi S. Genetic and epigenetic basis of Treg cell development and function: from a FoxP3centered view to an epigenome-defined view of natural Treg cells. Immunol Rev 2014;259:192-205.

68. Bridle BW, Chen L, Lemay CG, et al. HDAC inhibition suppresses primary immune responses, enhances secondary immune responses, and abrogates autoimmunity during tumor immunotherapy. Mol Ther 2013;21:887-94.

69. Shen L, Ciesielski M, Ramakrishnan S, et al. Class I histone deacetylase inhibitor entinostat suppresses regulatory $\mathrm{T}$ cells and enhances immunotherapies in renal and prostate cancer models. PLoS One 2012;7:e30815.

70. Beier UH, Wang L, Han R, et al. Histone deacetylases 6 and 9 and sirtuin-1 control Foxp3 + regulatory T cell function through shared and isoform-specific mechanisms. Sci Signal 2012;5:ra45.

71. Xiao H, Jiao J, Wang L, et al. HDAC5 controls the functions of Foxp3(+) T-regulatory and CD8(+) T cells. Int J Cancer 2016;138:2477-86.

72. Peng D, Kryczek I, Nagarsheth N, et al. Epigenetic silencing of TH1-type chemokines shapes tumour immunity and immunotherapy. Nature 2015;527:249-53.

73. Zou W, Chen L. Inhibitory B7-family molecules in the tumour microenvironment. Nat Rev Immunol 2008;8:467-77.

74. Pardoll DM. The blockade of immune checkpoints in cancer immunotherapy. Nat Rev Cancer 2012;12:252-64.

75. Zheng $\mathrm{H}$, Zhao $\mathrm{W}$, Yan $\mathrm{C}$, et al. HDAC inhibitors enhance T-cell chemokine expression and augment response to PD-1 immunotherapy in lung adenocarcinoma. Clin Cancer Res 2016;22:4119-32.

76. Motzer RJ, Escudier B, McDermott DF, et al. Nivolumab versus everolimus in advanced renal-cell carcinoma. $\mathrm{N}$ Engl J Med 2015;373:1803-13.

77. Rodriguez-Vida A, Hutson TE, Bellmunt J, et al. New treatment options for metastatic renal cell carcinoma. ESMO Open 2017;2:e000185.

78. Sharma P, Retz M, Siefker-Radtke A, et al. Nivolumab in metastatic urothelial carcinoma after platinum therapy (CheckMate 275): a multicentre, single-arm, phase 2 trial. Lancet Oncol 2017;18:312-22.

79. Bellmunt J, de Wit R, Vaughn DJ, et al. Keynote-045: open-label, phase III study of pembrolizumab versus investigator's choice of paclitaxel, docetaxel, or vinflunine for previously treated advanced urothelial cancer. J Immuno Ther Cancer 2016; 4:Abstract 470.

80. Balar AV, Castellano D, O'Donnell PH, et al. First-line pembrolizumab in cisplatin-ineligible patients with locally advanced and unresectable or metastatic urothelial cancer (KEYNOTE-052): a multicentre, single-arm, phase 2 study. Lancet Oncol 2017;18:1483-92.

81. Marciscano AE, Madan RA. Targeting the tumor microenvironment with immunotherapy for genitourinary malignancies. Curr Treat Options Oncol 2018;19:16.

82. Juengel E, Bhasin M, Libermann T, et al. Alterations of the gene expression profile in renal cell carcinoma after treatment with the histone deacetylase-inhibitor valproic acid and interferon-alpha. World J Urol 2011;29:779-86.

83. Gameiro SR, Malamas AS, Tsang KY, et al. Inhibitors of histone deacetylase 1 reverse the immune evasion phenotype to enhance T-cell mediated lysis of prostate and breast carcinoma cells. Oncotarget 2016;7:7390-402.

84. Sulek JE, Robinson SP, Petrossian AA, et al. Role of epigenetic modification and immunomodulation in a murine prostate cancer model. Prostate 2017;77:361-73.

85. Heninger E, Krueger TE, Thiede SM, et al. Inducible expression of cancer-testis antigens in human prostate cancer. Oncotarget 2016;7:84359-74.

86. Fradet Y, Picard V, Bergeron A, et al. Cancer-testis antigen expression in bladder cancer. Prog Urol 2006;16:421-8.

87. Hoffmann MJ, Muller M, Engers R, et al. Epigenetic control of CTCFL/BORIS and OCT4 expression in urogenital malignancies. Biochem Pharmacol 2006;72:1577-88.

Cite this article as: Grunewald CM, Schulz WA, Skowron MA, Hoffmann MJ, Niegisch G. Tumor immunotherapy-the potential of epigenetic drugs to overcome resistance. Transl Cancer Res 2018;7(4):1151-1160. doi: 10.21037/tcr.2018.06.24 